\title{
Mikroökonomie heute: ihre Bedeutung im Konzert der Methoden: Replik zum Kommentar von Viktor Vanberg
}

\author{
Joachim Weimann
}

Online publiziert: 6. März 2019

(C) List-Gesellschaft e.V. 2019

Zunächst möchte in Viktor Vanberg für seinen sehr ausführlichen Kommentar zu meinem Beitrag danken. Herr Vanberg nimmt zu vielen Punkten Stellung und nimmt dabei überwiegend (eigentlich stets) eine sehr kritische Haltung ein. Es würde viel zu viel Platz erfordern auf jeden einzelnen von ihm aufgeworfenen Punkt einzugehen. Deshalb werde ich mich in meiner Stellungnahme auf ein paar ausgesuchte, mir besonders wichtige Stellen beschränken.

Am Anfang möchte ich eine Klarstellung vornehmen. Meine Aufgabe war es, über die Rolle der Mikrotheorie in der heutigen Ökonomik zu berichten. Das bedeutet, dass ich mir die Frage vorgelegt habe, welche Funktion diese Theorie heute im Kontext der ökonomischen Forschung ausfüllt. Das zu erwähnen ist wichtig, weil deshalb die Auseinandersetzung mit anderen Bestandteilen der ökonomischen Forschungslandschaft notwendigerweise bestenfalls am Rande möglich war. Das gilt insbesondere für die Verhaltensökonomik, die in meinem Beitrag immer mal wieder erwähnt, aber nicht wirklich zentral behandelt wird. Das erfolgt in einem anderen Beitrag in diesem Band.

Herr Vanberg geht davon aus, dass ich den Versuch unternehme, aus der Mikrotheorie eine erfahrungswissenschaftliche Disziplin zu machen, um mir dann nachzuweisen, dass sie das nun mal nicht sei. Seiner Ansicht folgend, kann man eigentlich nur zu dem Schluss kommen, dass eine Theorie, die sich des Rationalmodells bedient, eigentlich überhaupt keine Daseinsberechtigung besitzt und deshalb auf den wissenschaftlichen Abfallhaufen gehört. Das sehe ich in der Tat anders und ich denke, dass ich die Bedeutung und den Wert einer normativen Theorie dargelegt habe.

This reply refers to the commentary article available online at https://doi.org/10.1007/s41025-01900142-7.

\footnotetext{
J. Weimann $(\bowtie)$

Otto-von-Guericke-Universität Magdeburg, Magdeburg, Deutschland

E-Mail: joachim.weimann@ovgu.de
} 
Davon aber abgesehen, halte ich auch die stillschweigend bei Herrn Vanberg immer mitschwingende Prämisse für falsch, dass die Mikrotheorie - weil sie ja normativ ist - keinerlei prognostische Kraft hat. Mikrotheoretische Modelle sagen dann etwas über die reale Welt aus, wenn ihre Annahmen (vor allem die rationalen Verhaltens) in dieser Welt erfüllt sind. Es ist eine ziemlich gewagte Hypothese, zu behaupten, dass sei nie der Fall. Ob die Rationalitätsannahme erfüllt ist oder nicht, lässt sich empirisch (zum Beispiel experimentell) überprüfen und es hat sich oft genug gezeigt, dass sich Menschen sehr wohl rational verhalten. Das gilt übrigens nicht nur für triviale Entscheidungen (kaufe ich den teuren oder den billigeren Zucker), sondern auch für durchaus komplexe Entscheidungssituationen.

In seiner Kritik geht Herr Vanberg von einem Wissenschaftsbild aus, in dem fein säuberlich zwischen den Methoden unterschieden wird, die helfen, empirisch gehaltvolle Hypothesen zu entwickeln und solchen, mit denen diese dann überprüft werden. Dieses Bild ist allerdings nicht mehr zeitgemäß. Es ist längst so, dass auch empirische Methoden existieren, mit denen ohne eine theoretisch begründete Hypothese zu besitzen, kausale Zusammenhänge nachgewiesen werden können. Es ist weiterhin in der experimentellen Forschung längst gängige Praxis, systematische Verhaltensbeobachtungen zu machen, die dann neue Hypothesen über Regularitäten des Verhaltens generieren können. Die Liste ließe sich fortsetzen. Es ist weiterhin schlicht und ergreifend falsch, wenn Herr Vanberg behauptet, dass sich aus mikroökonomischen Modellen keine testbaren Hypothesen ableiten lassen. In der experimentellen Literatur sind hunderte von spieltheoretischen Modellen überprüft worden, die die Rationalitätsannahme verwenden. Manche haben sich im Labor bewährt, andere nicht.

In Kapitel 4 geht es um die Frage, wie eine Theorie entwickelt werden kann, die sich dem Rationalmodell entgegenstellt und empirisch gehaltvolle Hypothesen formuliert. Wie bereits erwähnt, sind nicht alle Hypothesen, die Rationalität benutzen, per se nicht empirisch gehaltvoll. Aber darüber hinaus gibt es natürlich eine solche Theorie. Die Verhaltenstheorie versucht genau das, was Herr Vanberg fordert. Aber wie schon gesagt, sie darzustellen war nicht meine Aufgabe. Im Übrigen ist die vorgetragene Kritik nicht fair, denn sie verschweigt, dass ich an der zitierten Stelle vom „Neoklassischen Reparaturbetrieb“ rede und ausdrücklich darauf hinweise, dass es dazu eine Alternative gibt - eben die Verhaltensökonomik.

Im fünften Kapitel zitiert Herr Vanberg Kenneth Arrow und versucht damit nachzuweisen, dass das einfache Rationalmodell per se nicht das beschreiben kann, was Menschen tun, wenn sie Entscheidungen treffen. Beleg dafür sei, dass Menschen nicht Alternativen wählen, sondern Konsequenzen. Allerdings meint Arrow in diesem Zusammenhang, dass beides deshalb auseinanderfallen kann, weil die Welt unsicher ist und die Konsequenzen einer Entscheidung eben von unsicheren Umweltzuständen abhängen können. Weil das so ist, brauchen wir eine Theorie, die Entscheidungen unter Unsicherheit abbildet. Wir haben gleich zwei davon. Die Prospect Theorie und die SEU (subjective expected utility). Die erste ist verhaltensbasiert und die zweite ist ein axiomatischer Zugang. Viel relevanter wäre an dieser Stelle meines Erachtens der Hinweis auf die Existenz von Internalitäten gewesen, d.h. auf das Auseinanderfallen von Entscheidungsnutzen und Erfahrungsnutzen. 
Aber damit sind wir wieder in der Verhaltensökonomik - mit der sich ein Kollege in diesem Band befassen wird.

Auf den letzten Seiten seines Kommentars beschreibt Herr Vanberg, welche Art von Theorie seiner Einschätzung nach ein Gegenentwurf zur Mikrotheorie sein könnte. Kernidee ist, dass Menschen sich nicht strikt rational, sondern regelgebunden verhalten, weil das mittel- und langfristig für sie besser ist, als in jedem Einzelfall zu optimieren. In der Verhaltensökonomik wird - darauf weist Herr Vanberg auch hin - in dieser Richtung gedacht. Allerdings kommt das regelgebundene Verhalten dort in aller Regel als Heuristik oder systematische Verzerrung daher, die sich eben nicht als vorteilhaft erweisen, sondern zu systematische Fehler. Regeln, die im Sinne von Herrn Vanberg dem rationalen Kalkül überlegen sind, lassen sich dagegen kaum ausmachen. Allerdings weisen die Psychologen, die in der Verhaltensökonomik tätig sind (und nicht nur die) immer wieder darauf hin, dass nicht rationales Verhalten, dass den Automatismen des System 1 folgt (also des Teils unseres Gehirns, das für die schnellen Entscheidungen zuständig ist) unverzichtbar ist. Das wird kein Mikroökonom bezweifeln. Ob sich daraus aber Regeln ableiten lassen, die langfristig dem rationalen Kalkül überlegen sind, daran sind schon Zweifel angebracht. 